\title{
Difficultés associées à l'évaluation des vaccins contre le SRAS-CoV-2 pendant la pandémie de COVID-19
}

\author{
Bahaa Abu-Raya MD, Soren Gantt MD PhD, Manish Sadarangani BM BCh PhD
}

Citation : CMAJ 2020 August 24;192:E982-5. doi : 10.1503/cmaj.201237-f; diffusion hâtive le 9 juillet 2020

Voir la version anglaise de l'article ici : www.cmaj.ca/lookup/doi/10.1503/cmaj.201237

$\mathbf{L}$ a maladie à coronavirus 2019 (COVID-19), causée par le coronavirus du syndrome respiratoire aigu sévère 2 (SRASCoV-2), est à l'origine d'une pandémie qui évolue rapidement. Outre l'accès à des mesures non pharmacologiques permettant de réduire les risques de transmission, l'accès à un ou à plusieurs vaccins efficaces serait une ressource précieuse pour réduire le fardeau de la COVID-19. Plus de 140 vaccins potentiels contre le SRAS-CoV-2 sont en cours de développement et font l'objet d'études précliniques et d'essais cliniques ${ }^{1}$. En général, pour qu'un vaccin soit approuvé ou homologué par les organismes de réglementation, son innocuité et sa grande efficacité à prévenir la maladie visée chez une certaine population doivent être avérées. Or, la pandémie de COVID-19 pose des difficultés logistiques et scientifiques pour l'évaluation de l'efficacité des vaccins potentiels contre le SRAS-CoV-2, qui doit tenir compte du risque d'exposition de la population, de la susceptibilité au virus, des mesures d'éloignement sanitaire en place et de la région géographique. Par ailleurs, les vaccins contre le SRAS-CoV-2 doivent être évalués auprès des populations courant un risque accru de contracter une forme grave de COVID-192-4. Nous présentons ici les difficultés relatives à l'évaluation clinique des vaccins contre le SRAS-CoV-2, de même que des solutions potentielles.

\section{Habituellement, comment évalue-t-on les vaccins avant de les homologuer?}

Les vaccins à homologuer sont d'abord soumis à de petites études de phase 1 , qui visent à établir leur profil d'innocuité et leur capacité à entraîner une réponse immunitaire dirigée contre les antigènes administrés. Les vaccins qui répondent aux critères établis font ensuite l'objet d'études de phase 2, qui s'intéressent à leur innocuité, à leur immunogénicité et, parfois, à leur efficacité préliminaire chez des cohortes plus grandes. L'efficacité des vaccins est surtout évaluée dans les études de phase 3, qui cherchent à déterminer la réduction proportionnelle du taux d'infection prédéfini ou de maladie chez les participants vaccinés. L'efficacité représente le scénario le plus optimiste en ce qui concerne la protection conférée par le vaccin à l'étude, et elle doit être démontrée pour tout vaccin visant un nouvel agent pathogène avant son homologation par les organismes de régle-

\section{POINTS CLÉS}

- Les difficultés associées à l'évaluation, durant la pandémie, de vaccins potentiels contre le coronavirus du syndrome respiratoire aigu sévère 2 (SRAS-CoV-2) avant leur approbation ou leur homologation comprennent l'évolution rapide de l'exposition au virus et de l'immunité de la population, ainsi que les mesures d'éloignement sanitaire mises en place.

- Afin de bien évaluer l'efficacité des vaccins, les chercheurs devraient tenir compte de ces facteurs lors du calcul de la taille de l'échantillon étudié et sélectionner judicieusement les indicateurs de l'essai.

- Les vaccins contre le SRAS-CoV-2 doivent également être évalués au sein de populations courant un risque accru de développer une forme grave de la maladie à coronavirus 2019, comme les personnes âgées, les personnes noires et les personnes ayant plusieurs comorbidités.

- Étant donné la rapidité actuelle des processus de développement de vaccins contre le SRAS-CoV-2, il faut accorder une attention particulière à leur évaluation après l'homologation. Le risque de facilitation de l'infection par des anticorps, notamment, devrait être étroitement surveillé dans les années suivant la vaccination.

mentation ${ }^{2,3}$. Idéalement, l'évaluation de l'efficacité est réalisée dans le cadre d'études bien contrôlées (p. ex., essais randomisés et contrôlés à double insu). Les vaccins pour lesquels on réussit à démontrer une grande efficacité dans les essais cliniques et dont l'utilisation auprès d'une population est approuvée sont ensuite continuellement réévalués afin d'en vérifier l'innocuité et l'efficacité après leur homologation et le début de leur utilisation (dans des études de phase 4 , qui se penchent sur la réduction d'une maladie dans une population en contexte réel).

\section{Quels facteurs peuvent influencer l'évaluation des vaccins potentiels contre le SRAS-CoV-2?}

En général, des facteurs propres à l'hôte (âge, exposition génétique et environnementale, etc.) et au vaccin (antigènes sélectionnés, adjuvants, formule, type de vaccin et affaiblissement de l'immunité au fil du temps) influencent la réponse immunitaire d'une personne et, par conséquent, déterminent l'efficacité du 
Tableau 1: Facteurs populationnels nuisant à l'évaluation de l'efficacité des vaccins en cas d'infection endémique et d'infection pandémique, et facteurs propres à la maladie à coronavirus 2019

\section{Facteurs nuisant à la démonstration de l'efficacité des vaccins}

Transmission de référence dans la population (exposition) et caractère saisonnier de l'agent pathogène t

$\begin{array}{cc}\begin{array}{c}\text { Infection } \\ \text { endémique }\end{array} & \begin{array}{c}\text { Infection } \\ \text { pandémique }\end{array}\end{array}$

Connus

Connus

Évolution rapide; caractère saisonnier inconnu

\section{Facteurs propres à la COVID-19}

Éloignement sanitaire et autres interventions de santé publique
Immunité préexistante de la population contre l'agent pathogène (susceptibilité)
Connue Évolution rapide

Évolution rapide

Susceptibilité différentielle des sous-populations à l'infection ou à la maladie

Connue Émergente

\author{
Manque de données \\ séroépidémiologiques; \\ précision des tests \\ sérologiques; \\ méconnaissance de \\ l'intensité et de la durée de la \\ protection conférée par \\ l'immunité naturelle
De nombreux facteurs de risque ont été mis en évidence, mais les personnes âgées courent le risque le plus élevé de développer une infection grave; les jeunes enfants présentent rarement des complications et pourraient être moins susceptibles d'être infectés; facilitation de l'infection par des anticorps

\section{Stratégies}

Conception d'études flexibles permettant un nombre suffisant d'indicateurs (nombre d'infections ou d'hospitalisations); mise en évidence de facteurs de confusion potentiels attribuables aux mesures de prévention non vaccinales

\section{Réalisation de tests} sérologiques de référence chez les participants aux essais

Évaluation de l'efficacité des
vaccins et mesure des
indicateurs chez les personnes
âgées et les autres groupes à
risque accru; pour tous les
essais, exercer une surveillance
étroite et réaliser un suivi
prolongé afin de déceler toute
facilitation de l'infection par des
anticorps

Évaluation de l'efficacité des vaccins et mesure des indicateurs chez les personnes âgées et les autres groupes à risque accru; pour tous les essais, exercer une surveillance prolongé afin de déceler toute anticorps vaccin. Cependant, il est important de prendre en considération plusieurs autres facteurs parfois difficiles à quantifier, comme le degré de transmission de l'agent pathogène (exposition) et l'importance de l'immunité préexistante (susceptibilité) dans la population. Ces facteurs sont interreliés et varient selon que l'agent pathogène est endémique (généralement toujours présent dans une région géographique ou une population) ou pandémique (émergent et se transmettant partout sur la planète), comme le SRAS-CoV-2. L'exposition de référence d'une population à un agent pathogène donné, le caractère saisonnier de ce dernier et l'immunité de la population sont habituellement prévisibles en cas d'infection endémique, mais ce n'est pas le cas pour les infections pandémiques. Le tableau 1 résume les facteurs de confusion propres à la pandémie de COVID-19 pouvant nuire à l'évaluation de l'efficacité des vaccins potentiels contre le SRAS-CoV-2.

\section{Degré d'exposition au SRAS-CoV-2 et immunité}

Le schéma d'exposition au virus et l'immunité de la population durant la pandémie, qui évoluent constamment et rapidement, peuvent être des facteurs de confusion importants dans l'évaluation de l'efficacité des vaccins contre le SRAS-CoV-2, et ils devraient être pris en considération lors du calcul de la taille des échantillons des essais. Par exemple, si un essai est mené dans une population où l'incidence de la maladie est faible, ou si l'immunité conférée s'affaiblit considérablement quelque temps après la vaccination, il est possible qu'un vaccin hautement efficace ne semble pas offrir une protection significative si l'échantillon de l'essai est trop petit pour montrer une réduction significative du nombre de cas d'infection chez les participants vaccinés. Il faut donc porter une attention particulière au calcul de la taille de l'échantillon, et potentiellement recruter plus de participants dans les régions où la prévalence de la maladie est très faible. Par ailleurs, la transmission du SRAS-CoV-2 peut varier au sein d'un pays ou d'un pays à l'autre, et elle changera au fil de l'évolution de la pandémie; il faut donc en tenir compte lors de l'essai d'un vaccin dans plus d'une région.

La séropositivité, ou l'immunité de référence, de la population peut influencer l'immunogénicité du vaccin ou la susceptibilité de la population à l'agent pathogène, influençant ainsi les résultats d'évaluation. Par exemple, un vaccin hautement efficace pourrait ne pas apporter de bénéfices dans un milieu où la séroprévalence est élevée, puisqu'il n'augmenterait pas de façon significative la protection conférée par l'infection naturelle. Bien qu'elle ne soit pas un problème en début de pandémie, alors que la séropositivité de la population est faible ${ }^{4}$, la présence d'une immunité de référence élevée pourrait avoir un effet sur les résultats des essais 
sur les vaccins au fur et à mesure que progresse la situation et qu'augmente la séroprévalence (selon la population). Prenons à titre d'exemple un échantillon représentatif de la population de Genève (Suisse), dans lequel une augmentation des anticorps antiSRAS-CoV-2 a été observée en l'espace de 3 semaines consécutives en avril 2020, passant de $3,1 \%$ à $6,1 \%$, puis à $9,7 \%{ }^{5}$. Il va sans dire que la mesure de l'immunité préexistante dans les essais sur l'efficacité est importante, tout comme le sont l'inclusion, la vaccination et l'évaluation des participants séropositifs.

\section{Éloignement sanitaire et autres interventions de santé publique}

L'éloignement sanitaire s'est avéré efficace pour réduire la transmission du SRAS-CoV-2 $2^{6,7}$. Dans les milieux où la transmission est faible en raison des mesures d'éloignement mises en place, les bénéfices d'un vaccin très efficace pourraient être difficilement démontrables. Le calcul de la taille d'un échantillon devrait donc tenir compte de cette réalité. En outre, les interventions de santé publique sont susceptibles de varier selon l'évolution de la pandémie, ce qui compliquera les évaluations déjà en cours. II pourrait être nécessaire de faire preuve de flexibilité dans la conception des études pour avoir suffisamment d'indicateurs permettant de déterminer l'efficacité d'un vaccin en fonction de la fluctuation du taux de transmission à divers endroits.

\section{Quels préjudices associés au vaccin sont à prévoir?}

Bien qu'ils aient pour but de réduire le fardeau de la COVID-19, les vaccins contre le SRAS-CoV-2 pourraient en théorie entraîner une facilitation de l'infection par des anticorps ${ }^{8}$. Ce phénomène, observé dans des modèles animaux pour des vaccins contre le SRAS-CoV et d'autres coronavirus ${ }^{9}$, se caractérise par un faible titre d'anticorps ou par la présence d'anticorps neutralisants peu efficaces après la vaccination, ce qui facilite l'entrée ou la réplication du virus dans les cellules cibles et aggrave la maladie chez les personnes vaccinées l'ayant contractée. Comme l'a montré l'étude du vaccin CYD-TDV contre la dengue, la facilitation de l'infection par des anticorps après l'administration d'un vaccin peut avoir de graves répercussions sur les personnes vaccinées, mais aussi sur la confiance du public et le recours aux autres vaccins ${ }^{10,11}$. Elle pourrait se manifester après une vaccination contre le SRAS-CoV-2 au moyen de certains adjuvants, plateformes ou produits, mais pas tous. Fait important : il est possible qu'elle devienne apparente uniquement si un certain nombre de personnes ont été vaccinées et si la transmission de la COVID-19 est suffisamment élevée chez les personnes vaccinées pour générer un fardeau important. Il est également possible qu'elle accompagne l'affaiblissement de la réponse immunitaire après quelques années, ou qu'elle soit associée à des changements dans le génotype viral survenant au fil de temps ${ }^{12}$. Nous pensons que le risque de facilitation de l'infection par des anticorps devrait être activement surveillé pendant plusieurs années en portant attention à toute diminution des titres d'anticorps ou variation dans les souches virales en circulation (tableau 1).

\section{Pourquoi la détermination réfléchie de la population à l'étude et des indicateurs des essais est-elle importante?}

Comme tout vaccin, les vaccins contre le SRAS-CoV-2 sont d'abord testés auprès d'adultes volontaires en bonne santé. Or, il a été montré que la COVID-19 affecte plus gravement les personnes âgées, les personnes noires et les personnes ayant plusieurs comorbidités ${ }^{13,14}$. Par conséquent, les résultats provenant des populations à faible risque pourraient ne pas être représentatifs des possibles bénéfices et préjudices chez les populations à risque accru. Il se peut également qu'un vaccin ne confère pas une immunité stérilisante (définie comme un état immunitaire prévenant l'infection par le virus après la vaccination), ce qui signifie que les personnes vaccinées pourraient éventuellement développer une forme bénigne de la maladie. Dans ce cas, la vaccination aurait une plus grande incidence sur la prévention de la forme grave de la maladie que sur la prévention des symptômes bénins ou de l'infection par le virus. Ces éléments devraient être pris en considération lors du choix des indicateurs cliniques utilisés dans le cadre des essais évaluant l'efficacité des vaccins contre le SRAS-CoV-2. Par exemple, si un essai a pour but de montrer qu'un vaccin réduit efficacement le nombre de cas et d'issues graves de la COVID-19, des indicateurs comme le nombre d'hospitalisations, le nombre d'admissions en soins intensifs, le besoin de soutien respiratoire et le nombre de décès pourraient être considérés comme des résultats primaires, mais un faible taux d'événements graves, selon le taux global d'infection, pourrait nécessiter le recrutement d'un plus grand nombre de participants.

Il est également important que les vaccins soient testés auprès des populations courant un risque accru de développer une forme grave de COVID-19, comme les personnes âgées, les travailleurs de la santé, les personnes noires et les personnes ayant des problèmes de santé prédisposants ${ }^{15}$. Il s'agit là d'un élément essentiel qui renseignera les responsables des orientations politiques sur les populations qui profiteraient le plus de la vaccination et qui devraient passer en priorité en cas de pénurie de vaccin.

\section{Quelles autres difficultés sont associées à la mise au point des vaccins contre le SRAS-CoV-2?}

\section{Difficultés en lien avec la réglementation}

La mise au point de vaccins destinés aux humains prend généralement de 10 à 15 ans, de la conception préclinique à l'homologation. Toutefois, étant donné l'urgence actuelle, le développement et l'approbation des vaccins contre le SRASCoV-2 devront être considérablement accélérés, prenant idéalement quelques mois plutôt que des années. Un lourd fardeau pourrait donc être imposé aux organismes de réglementation, qui devront traiter les demandes beaucoup plus rapidement qu'à l'habitude. 


\section{Difficultés en lien avec l'innocuité}

Dans les 10 dernières années, de nouvelles technologies pour l'administration des vaccins ont vu le jour (p. ex., vaccins à base d'ADN ou d'ARN). Bien qu'elles n'aient jamais servi à la conception des vaccins homologués jusqu'ici, ces technologies ont été utilisées pour élaborer certains des vaccins contre le SRAS-CoV-2 qui sont rapidement passés aux essais cliniques ${ }^{15}$. L'innocuité de ces vaccins potentiels devrait donc être évaluée avec soin. Pour ce faire, il faudra continuellement rester à l'affût des effets indésirables fréquents et des effets indésirables rares survenant chez un grand nombre de personnes vaccinées, tant au cours des essais cliniques qu'après la commercialisation des vaccins.

\section{Quelles occasions et difficultés organisationnelles particulières accompagnent les essais sur les vaccins contre le SRAS-CoV-2?}

\section{Logistique de l'évaluation de plusieurs vaccins}

L'étude simultanée de plusieurs vaccins dans une même population peut poser problème en cas de capacités d'évaluation limitées. Cette difficulté peut être surmontée en concevant une étude flexible qui facilite l'ajout ou le retrait de vaccins au fur et à mesure que des données sur leur innocuité, leur immunogénicité et leur efficacité sont rendues disponibles. Ce type d'approche est actuellement employé dans le cadre d'essais cliniques évaluant divers traitements pharmacologiques contre la COVID-19 (p. ex., l'essai Solidarity, organisé par l'Organisation mondiale de la Santé et ses partenaires ${ }^{16}$ ). De plus, on procède actuellement à la création d'infrastructures qui permettent de faire appel à des équipes habituées d'évaluer des médicaments autres que des vaccins, et qui soutiennent la mise sur pied rapide de nouveaux sites d'essai, tout en offrant une formation ciblée aux chercheurs.

\section{Collaborations nationales et internationales}

La formation de consortiums nationaux et internationaux pour les essais des vaccins contre le SRAS-CoV-2 favorisera la collaboration des parties prenantes d'un même pays ou de divers pays. Parmi les exemples se trouve le Réseau canadien de recherche sur l'immunisation, qui rassemble des chercheurs canadiens menant des projets collaboratifs en vaccinologie. Pour que l'évaluation des vaccins soit équitable, les échantillons des divers sites d'étude doivent représenter l'entièreté de la population locale, et les études doivent être menées dans des pays à revenu faible, intermédiaire et élevé. Comme la demande pour des vaccins efficaces sera grande, il faudra absolument fixer des critères assurant une distribution équitable après l'homologation.

\section{Comment l'évaluation des vaccins devrait-elle être effectuée une fois ceux-ci homologués?}

L'évaluation posthomologation de l'efficacité d'un vaccin administré à la population repose généralement sur des études rétrospectives cas-témoins, ou sur des études écologiques ou observationnelles. Ces études devraient comprendre des populations dont le risque d'exposition, l'immunité préexistante (si elle est connue), la provenance géographique et les caractéristiques de référence (âge, comorbidités, etc.) varient. Elles pourraient également tenir compte du temps écoulé depuis la vaccination pour évaluer l'efficacité des vaccins contre le SRAS-CoV-2 au fil du temps, et ainsi déterminer s'il y a affaiblissement de l'immunité à la longue.

\section{Conclusion}

Nous avons présenté les difficultés propres à l'évaluation des vaccins contre le SRAS-CoV-2 durant la pandémie. Ces difficultés sont associées à divers facteurs : changement rapide de l'exposition au virus; évolution de l'immunité de la population; effets des mesures d'éloignement sanitaire locales; vitesse à laquelle les vaccins potentiels sont mis au point et testés; considérations relatives à l'innocuité des technologies non testées jusqu'ici; et besoin de décider quels indicateurs sont importants, de déterminer quelles personnes sont les plus touchées et de repérer les problèmes d'équité en ce qui concerne l'accès aux vaccins approuvés. De plus, comme l'efficacité des vaccins pourrait être difficile à démontrer chez certaines populations, les chercheurs devraient mener une analyse approfondie de la réponse immunitaire protectrice (humorale et cellulaire) après l'infection naturelle et après la vaccination, l'établissement de corrélations pouvant guider la mise au point et l'évaluation des vaccins.

\section{Références}

1. Draft landscape of COVID-19 candidate vaccines. Geneva: World Health Organization; 2020. Disponible : www.who.int/publications/m/item/draft-landscape -of-covid-19-candidate-vaccines (consulté le 26 mai 2020).

2. Clemens J, Brenner R, Rao M, et al. Evaluating new vaccines for developing countries. Efficacy or effectiveness? JAMA 1996;275:390-7.

3. Weinberg GA, Szilagyi PG. Vaccine epidemiology: efficacy, effectiveness, and the translational research roadmap. J Infect Dis 2010;201:1607-10.

4. Sood N, Simon P, Ebner P, et al. Seroprevalence of SARS-CoV-2-Specific Antibodies Among Adults in Los Angeles County, California, on April 10-11, 2020. JAMA 2020;323:2425-7.

5. Stringhini S, Wisniak A, Piumatti G, et al. Repeated seroprevalence of antiSARS-CoV-2 IgG antibodies in a population-based sample from Geneva, Switzerland. medRxiv 2020 May 6. doi: 10.1101/2020.05.02.20088898.

6. Shoukat A, Wells CR, Langley JM, et al. Projecting demand for critical care beds during COVID-19 outbreaks in Canada. CMAJ 2020;192:E489-96.

7. Tuite AR, Fisman DN, Greer AL. Mathematical modelling of COVID-19 transmission and mitigation strategies in the population of Ontario, Canada. CMAJ 2020; 192:E497-505

8. Hotez PJ, Corry DB, Bottazzi ME. COVID-19 vaccine design: the Janus face of immune enhancement. Nat Rev Immunol 2020;20:347-8.

9. Tseng CT, Sbrana E, Iwata-Yoshikawa N, et al. Immunization with SARS coronavirus vaccines leads to pulmonary immunopathology on challenge with the SARS virus. PLoS One 2012;7:e35421.

10. Halstead SB. Critique of World Health Organization recommendation of a dengue vaccine. J Infect Dis 2016;214:1793-5.

11. Philippines gripped by dengue vaccine fears. British Broadcasting Corporation 2018 Feb. 3. Disponible : www.bbc.com/news/world-asia-42929255 (consulté le 26 mai 2020). 
12. Korber B, Fischer WM, Gnanakaran S, et al. Spike mutation pipeline reveals the emergence of a more transmissible form of SARS-CoV-2. bioRxiv 2020 Apr. 30. doi: $10.1101 / 2020.04 .29 .069054$.

13. Yancy CW. COVID-19 and African Americans. JAMA 2020;323:1891-1892.

14. Onder G, Rezza G, Brusaferro S. Case-fatality rate and characteristics of patients dying in relation to COVID-19 in Italy. JAMA 2020 Mar. 23 [Cyberpublication avant impression]. doi: 10.1001/jama.2020.4683.
15. Amanat F, Krammer F. SARS-CoV-2 vaccines: status report. Immunity 2020;52: 583-9.

16. Cheng MP, Lee TC, Tan DHS, et al. Generating randomized trial evidence to optimize treatment in the COVID-19 pandemic. CMAJ 2020;192:E405-7.
Intérêts concurrents : Soren Gantt reçoit des honoraires de consultant de Moderna, de Merck, de GlaxoSmithKline et de Curevo; du financement pour la recherche sur les vaccins de Merck, de GlaxoSmithKline et de VBI Vaccines; et du financement pour l'essai d'un vaccin contre la COVID-19 de Symvivo. Soren Gantt est également membre ayant droit de vote du Comité consultatif national de l'immunisation. Manish Sadarangani a pris part à des projets de recherche financés par Pfizer, Merck, Seqirus, Sanofi Pasteur, VBI Vaccines et GlaxoSmithKline, et a reçu du financement pour l'essai d'un vaccin contre la COVID-19 de Symvivo. Tous les fonds ont été versés à son établissement; il n'a reçu aucun honoraire personnel. Aucun autre intérêt concurrent n'a été déclaré.

Cet article a été révisé par des pairs.

Affiliations : Centre d'évaluation de vaccins, Institut de recherche de l'Hôpital pour enfants de la Colombie-Britannique, et Division d'infectiologie, Département de pédiatrie, Université de la Colombie-Britannique, Vancouver (Colombie-Britannique)

Collaborateurs : Tous les auteurs ont contribué à l'élaboration et à la conception de l'étude. Bahaa Abu-Raya a rédigé la première version du manuscrit. Tous les auteurs ont révisé de façon critique le manuscrit; ils ont donné leur approbation finale pour la version destinée à être publiée, et assument l'entière responsabilité de tous les aspects du travail.

Remerciements : Bahaa Abu-Raya est titulaire d'une bourse d'études supérieures du Canada Vanier des Instituts de recherche en santé du Canada. Manish Sadarangani est titulaire d'une bourse salariale de la Fondation de l'Hôpital pour enfants de la ColombieBritannique, du Programme canadien de cliniciens-chercheurs en santé de l'enfant et de la Fondation Michael Smith pour la recherche en santé.

Correspondance : Bahaa Abu-Raya, baburaya@bcchr.ubc.ca 